\title{
Diagnosis of Liver Tumor from CT Images Using Fast Discrete Curvelet Transform
}

\author{
S.S. Kumar \\ Department of EIE, \\ Noorul Islam University, \\ Kumaracoil-629180, India
}

\author{
Dr R.S. Moni \\ Department of EEE, \\ Noorul Islam University, \\ Kumaracoil-629180, India
}

\begin{abstract}
In this paper, a novel feature extraction scheme is proposed, based on multiresolution fast discrete curvelet transform for computer-aided diagnosis of liver diseases. The liver is segmented from CT images using adaptive threshold detection and morphological processing. The suspected tumour region is extracted from the segmented liver using FCM clustering. The textural information obtained from the extracted tumour using Fast Discrete Curvelet Transform (FDCT) is used to train and classify the liver tumour into hemangioma and hepatoma employing artificial neural network classifier. A comparison with a similar algorithm based on Wavelet texture descriptors shows that using FDCT based texture features significantly improves the classification rate of liver tumours from CT scans.
\end{abstract}

\section{Keywords}

Liver Tumour, FCM technique, Texture analysis, Fast Discrete Curvelet Transform

\section{INTRODUCTION}

Liver diseases are considered seriously because of the liver's vital importance to human beings. There are two classes of liver tumours: benign and malignant. The benign tumour of the liver considered for this work is cavernous hemangioma and the malignant tumour considered is hepatoma (hepatocellular carcinoma) [1]. The computer aided diagnostic (CAD) system consists of three parts. Segmentation of liver and tumour from abdominal CT image, extraction of features and classification of liver diseases using a classifier. Texture is a commonly used feature in the analysis and interpretation of tissues in Medical images. One way of characterizing texture is by calculating a set of local statistical properties of the pixel grey level intensity, measuring variations on a surface such as smoothness, coarseness and regularity. In CAD based liver disease classification, texture features are extracted using co-occurrence matrices, run-length statistics, Law's texture and statistical moments [2]-[6]. A drawback of these texture analysis schemes is that the image is analyzed at only one single scale. Though multi-resolution analysis like Gabor filtering and wavelets were successfully used [7]-[9], they have limited directionality and decomposition. Recently, the finite contourlet and curvelet transforms have emerged as new multi-resolution analysis tools. These tools have better directional decomposition capabilities than wavelets [10]. In this paper a CAD system is proposed for classification of liver tumours from CT scans using fast discrete curvelet transform based feature extraction.

The paper is structured as follows: Section 2 describes in detail the automatic segmentation of liver and tumour from abdominal CT images. Section 3 describes the FDCT texture analysis, Section 4 describes the artificial neural network classifier; implementation results are presented in Section 5 and conclusion in Section 6.

\section{LIVER AND TUMOUR EXTRACTION}

Segmenting the liver alone from the abdominal CT image is difficult due to the fact that the image includes other organs like kidney, spleen, pancreas etc very close to the liver. A fixed threshold cannot be used for liver segmentation because the liver intensity differs according to the patient slice and the CT machine. Therefore, a system is developed to extract the liver automatically with adaptive threshold detection. For this a histogram of the CT image is drawn and analyzed. The highest pitch excluding the background and bone values, represent the middle intensity of the liver region. Certain margin is included within the intensity range of the liver region to accommodate any variation in the liver region pixels. Based on these processes, the proper intensity range corresponding to the liver region can be adaptively obtained for each slice. The pixels in the determined range of intensity are extracted. Then morphological processing is used to preserve the liver structure and remove the fragments of other organs using the conditions like area and location of liver [11]. Thereafter the tumour is extracted from the segmented liver image using Fuzzy $\mathrm{C}$ Means clustering which can determine the threshold regardless of a changing intensity. FCM minimizes the object function through the iterative optimization of the membership function based on the similarity between the data and the center of a cluster. It varies the threshold between clusters through an iterative process. The threshold is determined appropriately for every slice and the tumour region can be successfully extracted [12]. The tumour region is outputted for further analysis.

\section{FDCT BASED FEATURE EXTRACTION}

Candes and Donoho introduced a new multiscale transform named Curvelet transform which is designed to represent edges and other singularities along curves much more efficiently than the traditional transforms [10]. There are two separate Discrete Curvelet Transform (DCT) algorithms introduced by Candes, 
Donoho and Demanet [13]. The first algorithm is the unequispaced FFT transform, where the curvelet coefficients are found by irregularly sampling the Fourier coefficients of an image. The second algorithm is the wrapping transform, which uses a series of translation and a wrap around techniques. The wrapping FDCT is more intuitive and has less computation time. So it is used for extraction of features from tumour image. The wrapping discrete curvelet transform is implemented using the following steps. (i) FFT of the tumour image is taken and the resulting Fourier samples is divided into collection of digital corona tiles as shown in "Figure.1". (ii) For each corona tile, the tile is translated to the origin as shown in "Figure.2". (iii) The parallelogram shaped support of the tile is wrapped around a rectangle centered at the origin as shown in "Figure 3". (iv) The Inverse FFT of the wrapped support is determined and finally the resulting curvelet array is added to the collection of curvelet coefficients.

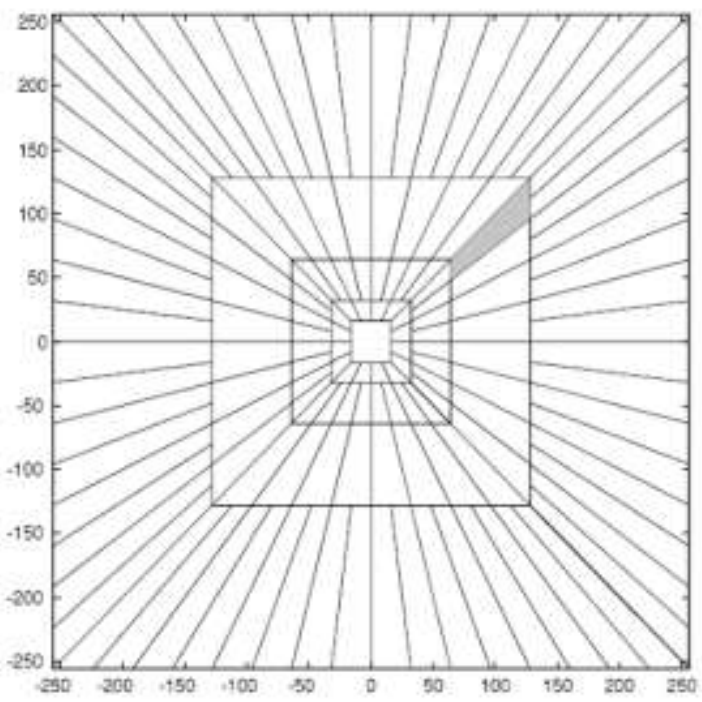

Figure 1. Digital corona of the Frequency Domain

Fast discrete curvelet transform can be calculated to various resolutions or scales and angles. Two parameters are involved in the digital implementation of the curvelet transform: number of resolutions and number of angles at the coarsest level [11]. In the proposed method, the tumour image is decomposed into three scales using real-valued curvelets. The number of second coarsest level angles used is 16. This results in 1 subband at finest level $(\mathrm{L}=1), 16$ subbands at second coarsest level $(\mathrm{L}=2)$ and again 1 subband corresponding to last coarsest level $(\mathrm{L}=3)$. The features like mean, standard deviation, energy and entropy for the obtained subbands are calculated and stored in a feature vector. This feature vector is fed as the input to the classifier for the purpose of training and testing.

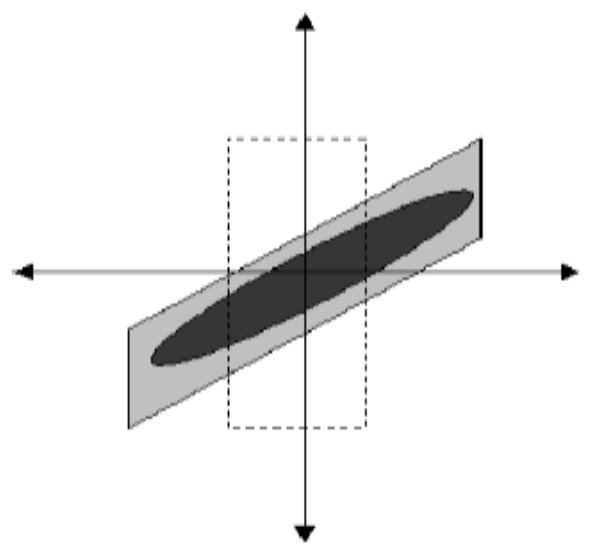

Figure 2. Support of wedge before wrapping

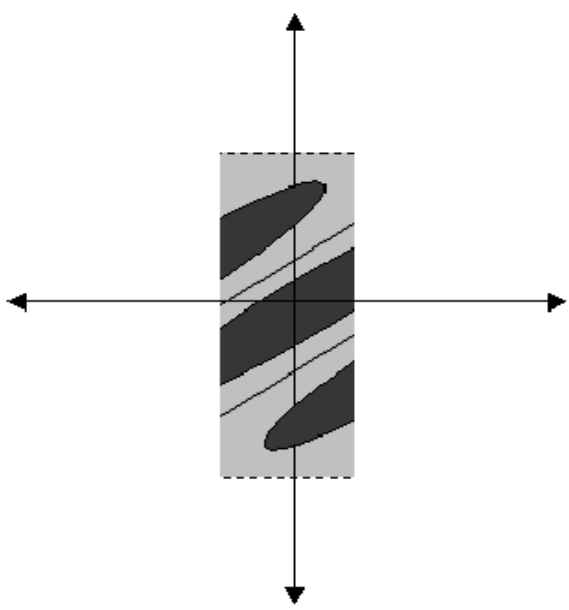

Figure 3. Support of wedge after wrapping

\section{CLASSIFICATION}

Artificial neural networks have proven themselves as proficient classifiers and are particularly well suited for tumour classification [14], [15]. Hence, it is decided to use a pattern recognition network, which is a feed-forward network with tansigmoid transfer functions in both the hidden layer and the output layer. The network has two output neurons, as there are two classes associated with each input vector. The performance of the classifier is evaluated by calculating accuracy, selectivity and specificity from the obtained confusion matrix. An ROC curve is also plotted, which is a plot of the true positive rate (Sensitivity) versus the false positive rate ( 1 - Specificity) as the threshold is varied. Here, Sensitivity=True Positive/Total Positive, Specificity $=$ True Negative/Total Negatives and Accuracy $=$ (True Positive + True Negatives)/Total Samples.

\section{IMPLEMENTATION AND RESULTS}

In this work, the liver tumour is classified as benign: hepatoma and malignant: hemangioma. All the CT images used in the experiment are in DICOM format and were acquired from Doctor's Scans; Marthandam .The sizes of the images used are $256 \times 256$ and were collected from different hemangioma 
patients and hepatoma patients. From these patients, 50 hemangioma images and 40 hepatoma images are considered for this work. The input dataset of 90 images are grouped in to two groups; a training set and a testing set with 45 datasets each.

Figure 4, shows the abdominal CT scan image of a patient with liver tumour. The liver is located along the left half of the image. Histogram of the image with liver intensity marked is shown in Figure 5. With the help of adaptive threshold detection and morphological operations, the segmented liver from CT image is shown in Figure 6 and Figure 7. The tumour is extracted from the segmented liver image by applying FCM Clustering technique and is shown in Figure 8.

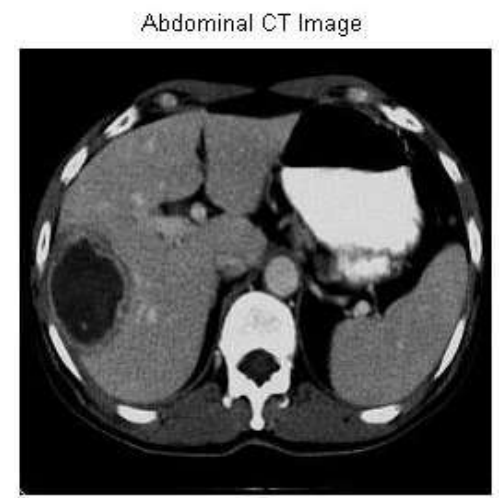

Figure 4. The Abdominal CT image

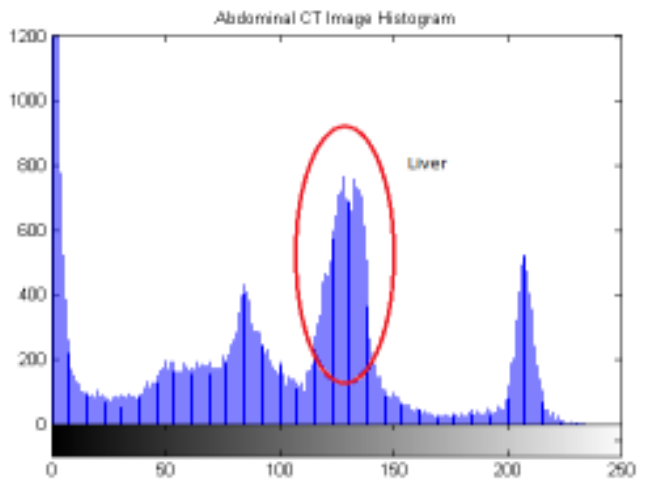

Figure 5. Histogram showing Liver Intensity marked

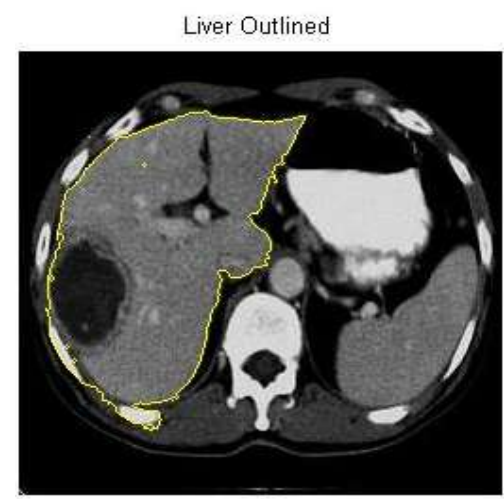

Figure 6. Outlined Liver

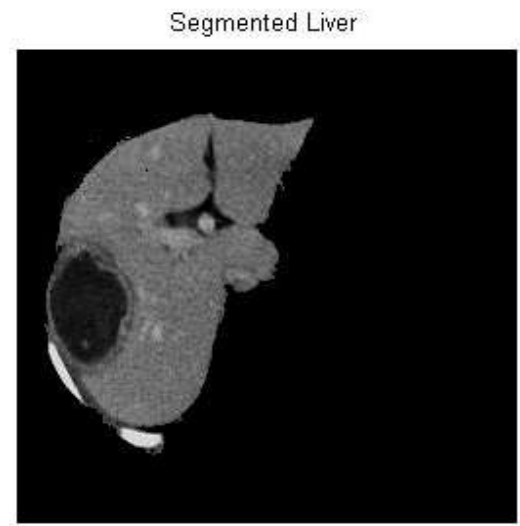

Figure 7. Segmented Liver

Segmented Tumor

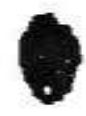

Figure 8. Extracted Tumour

Figure 9, shows the FDCT coefficients derived using FDCT via wrapping. The low frequency coefficients are stored at the centre of the display. The cartesian concentric corona shows the coefficients at different scales; the outer coronae correspond to higher frequencies. There are four strips associated to each corona, corresponding to the four cardinal points; these are further subdivided in to 16 angular panels. Each panel represent coefficients at a specified scale and along the orientation suggested by the position of the panel.

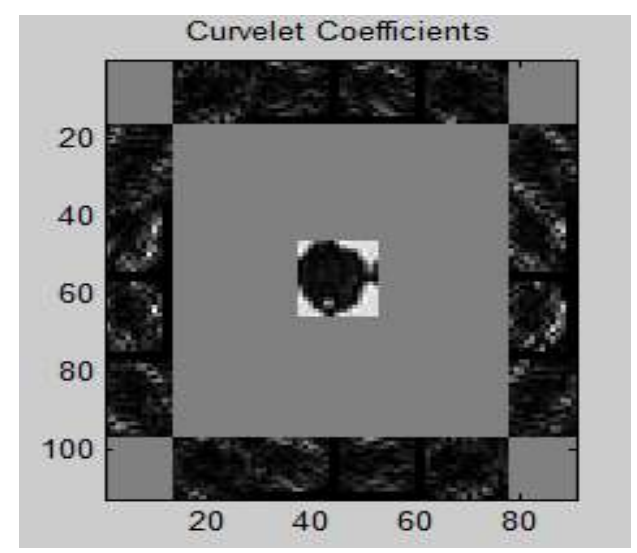

Figure 9. FDCTcoefficients 
The mean, standard deviation, energy and entropy were calculated for each subbands and arranged in the feature vector $\mathrm{f}$. This texture feature vectors derived from the training and testing dataset is used for classification with neural networks. The pattern recognition tool is used for classification of tumour as hemangioma and hepatoma. A set of 25 hemangioma and 20 hepatoma were used for training the network, and another set of 25 hemangioma and 20 hepatoma were used for testing the classifier. The confusion matrix of FDCT based classification is shown in Table 1.

In Table 1, class 1 represents Hepatoma and class 2 represents Hemangioma. The diagonal cells show the number of cases that were correctly classified, and the off-diagonal cells show the misclassified cases. The total percent of correctly classified cases is $93.3 \%$ and the total percent of misclassified cases is $6.7 \%$. 18 of the total 20 hepatoma cases were correctly classified as hepatoma, while 2 of them were misclassified as hemangioma. 24 of the 25 hemangioma cases used for testing were correctly classified as hemangioma, while 1 of them was misclassified as hepatoma.

Table 1. Confusion Matrix for FDCT based method

\begin{tabular}{|c|c|c|}
\cline { 2 - 3 } \multicolumn{1}{c|}{} & \multicolumn{2}{c|}{ Target Class } \\
\hline Output Class & Class 1 & Class 2 \\
\hline Class 1 & $\mathbf{1 8}$ & $\mathbf{1}$ \\
\hline Class 2 & $\mathbf{2}$ & $\mathbf{2 4}$ \\
\hline
\end{tabular}

In order to evaluate the performance of the proposed method, its performance measures were compared with the measures derived from wavelet based method. For this the feature vectors were derived from the tumour images using wavelet transform with three levels of decomposition and classified using the same neural classifier. The confusion matrix is given in Table. 2 .

The ROC curve is a plot of the true positive rate (sensitivity) versus the false positive rate (1 - specificity) as the threshold is varied. Since the test shows points in the upper-left corner $(0,1)$, it is concluded that the network performs well in FDCT based classification than the wavelet based method.

In Table 2, class 1 represents Hepatoma and class 2 represents Hemangioma. The diagonal cells show the number of cases that were correctly classified, and the off-diagonal cells show the misclassified cases. The total percent of correctly classified cases is $88.9 \%$ and the total percent of misclassified cases is $11.1 \%$. 16 of the total 20 hepatoma cases were correctly classified as hepatoma, while 4 of them were misclassified as hemangioma. 24 of the 25 hemangioma cases used for testing were correctly classified as hemangioma, while 1 of them was misclassified as hepatoma.

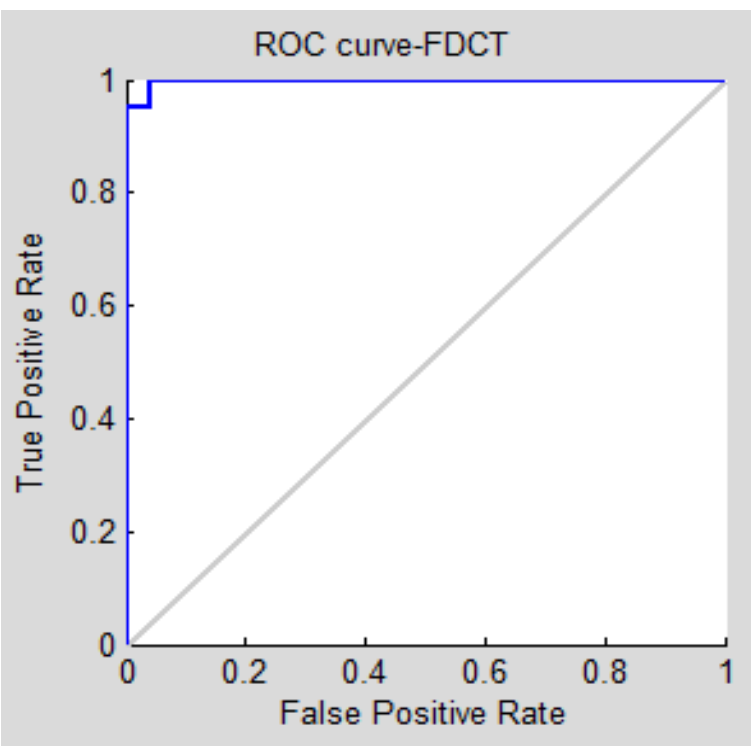

Figure 10. ROC for FDCT based method

Table 2. Confusion Matrix for Wavelet based method

\begin{tabular}{|c|c|c|}
\cline { 2 - 3 } \multicolumn{1}{c|}{} & \multicolumn{2}{c|}{ Target Class } \\
\hline Output Class & Class 1 & Class 2 \\
\hline Class 1 & $\mathbf{1 6}$ & $\mathbf{1}$ \\
\hline Class 2 & $\mathbf{4}$ & $\mathbf{2 4}$ \\
\hline
\end{tabular}

Table 3, gives the various performance measures calculated from the confusion matrix of both FDCT and wavelet based methods. The results shows that the FDCT-based texture features outperform the wavelet-based descriptors in classifying liver tumours from CT images. The accuracy of classification of tumours using FDCT based texture features extraction is $93.3 \%$ while it is only $88.9 \%$ using wavelet based method. The graphical comparisons of accuracies of both methods are shown in Figure 12. Since accuracy reflects both the sensitivity and specificity in relation to each other, it is selected to determine the overall correctness of the classifier. The Figure 12 shows that the FDCT based disease classification has better accuracy than wavelet based method. The accuracy has improved by $4.4 \%$ using FDCT method. This is because the fast discrete curvelet transform is able to capture multi-directional features as opposed to wavelet transform which focuses mainly on horizontal, vertical and diagonal features only. 


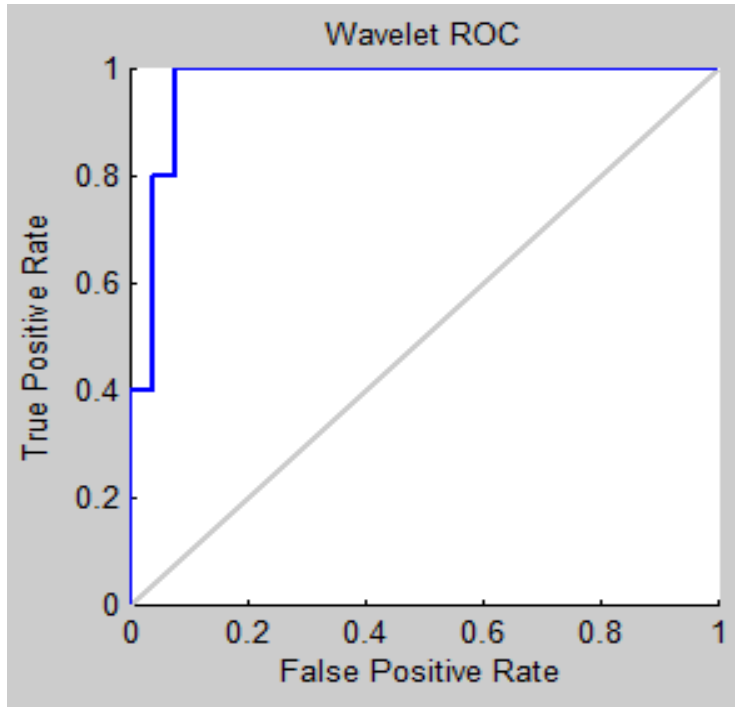

Figure 11. ROC for Wavelet based method

Table 3. Performance Measures

\begin{tabular}{|l|l|l|l|l|}
\hline \multirow{2}{*}{ Type } & \multicolumn{4}{|c|}{ Measures } \\
\cline { 2 - 5 } & Accuracy & Specificity & Sensitivity & Precision \\
\hline FDCT & $93.3 \%$ & $96 \%$ & $90 \%$ & $94.73 \%$ \\
\hline Wavelet & $88.88 \%$ & $96 \%$ & $80 \%$ & $94.11 \%$ \\
\hline
\end{tabular}

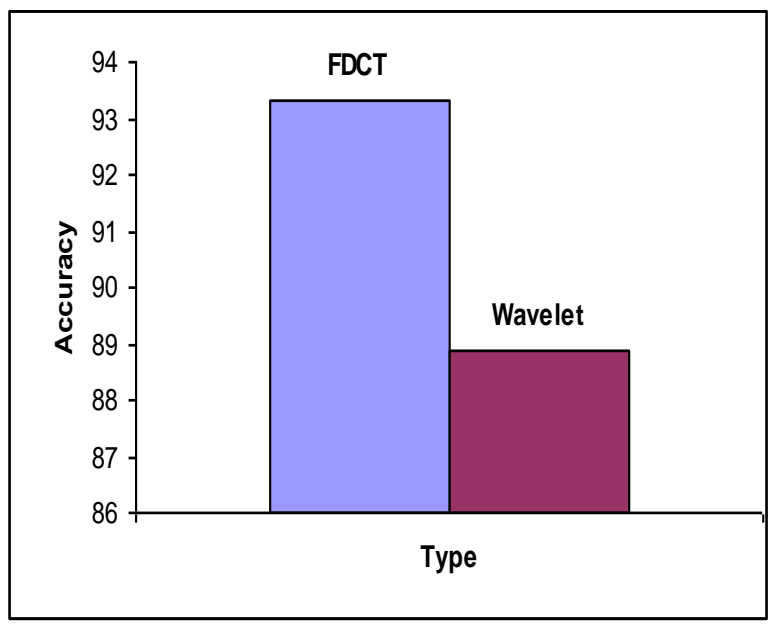

Figure 12.ClassificationPerformance

\section{CONCLUSION}

In this paper, the design and implementation of a CAD system consisting of liver and tumour segmentation, feature extraction and classification module is presented characterizing the CT liver tumour as hemangioma and hepatoma. The experiment results show that the classification accuracy of FDCT based feature extraction and classification is higher than the wavelet based method. The performance measures can be increased by increasing the number of samples used. The proposed system can be extended for diagnosis of other types of liver diseases

\section{REFERENCES}

[1] Dushyant V. Sahani, Sanjeeva P. Kalva, "Imaging the Liver" The Oncologist, 2004, 9: 385-397.

[2] S. Poonguzhali, B. Deepalakshmi and G. Ravindran, "Optimal Feature Selection and Automatic Classification of Abnormal Masses in Ultrasound Liver Images", IEEE ICSCN 2007, MIT Campus, Anna University, Chennai, India. Feb. 22-24, 2007. Pp.503-506.

[3] Yasser M. Kadah, Aly A. Farag, Jacek M. Zaruda, Ahmed M. Badawi, and Abou-Bakr M. Youssef., "Classification Algorithms for Quantitative Tissue Characterization of Diffuse Liver Disease from Ultrasound Images," IEEE transactions on Medical Imaging Vol 15, No 4, pp 466-477, August 1996.

[4] E-Liang Chen, Pau-CHoo Chung, Ching-Liang Chen, HongMing Tsai and Chein I Chang, "An Automatic Diagnostic system for CT Liver Image Classification", IEEE Transactions Biomedical Engineering, vol 45, no. 6, pp. 783-794, June 1998.

[5] Pavlopoulos.S, Kyriacou.E, Koutsouris.D, Blekas.K, Stafylopatis.A, Zoumpoulis.P, "Fuzzy Neural NetworkBased Texture Analysis of Ultrasonic Images," IEEE Engineering in Medicine and Biology, pp 39-47, Feb 2000.

[6] Yu-Len Huang, Jeon-Hor Chen1, Wu-Chung Shen1 "Computer-Aided Diagnosis of Liver Tumours in Nonenhanced CT Images" Department of Computer Science and Information Engineering, Tunghai University,Mid Taiwan, Journal of Medical Physics, Vol. 9, pp. 141-150, 2004.

[7] Chien-Cheng Lee, and Sz-Han Chen, "Gabor Wavelets and SVM Classifier for Liver Diseases Classification from CT Images", 2006 IEEE International Conference on Systems, Man, and Cybernetics October 8-11, 2006, Taipei, Taiwan

[8] Aleksandra Mojsilovic, Miodrag Popovic, Srdjan Markovic and Miodrag Krstic, "Characterization of Visually Similar Diffuse Diseases from B-Scan Liver Images using Non Separable Wavelet Transform", IEEE Transactions on Medical Imaging, vol. 17, no. 4, pp. 541-549, Aug. 1998.

[9] Semler, L., Dettori, L., \& Furst, J. Wavelet-Based Texture Classification of Tissues in Computed Tomography. Proceedings of the 18th IEEE International Symposium on Computer-Based Medical Systems, 265-270. (2005).

[10] David L. Donoho \& Mark R. Duncan, Digital Curvelet Transform: Strategy, Implementation and Experiments, Stanford University, November, 1999. 
[11] Robert M Haralick, Stanely R Sternberg and Xinhua Zhuang, "Image analysis using Mathematical Morphology", IEEE Transactions on Pattern Analysis and Machine Intelligence, vol. PAMI-9, no. 4, pp.532-549, July, 1987.

[12] Weijie Chen, Maryellen L. Giger, and Ulrich Bick,” A fuzzy c-means (FCM)-based approach for computerized segmentation of breast lesions in dynamic contrast-enhanced MR images." Academic Radiology, Vol 13, No 1, January 2006.

[13] Candes, E., Demanet, L., Donoho, D., \& Ying, L. "Fast Discrete Curvelet Transforms", Technical Report, Cal Tech, (2005).
[14] H. Sujana, S. Swarnamani, and S. Suresh, "Application of artificial neural networks for the classification of liver lesions by image texture parameters," Ultrasound in Medicine and Biology, vol. 22, no. 9, pp. pp. 1177-1181, Sept. 1996.

[15] M. Gletsos, et al., "A Computer-aided diagnostic system to characterize CT focal liver lesions: design and optimization of a neural network Classifier," IEEE Trans. Inform. Techno. Bio med., vol. 7, no. 3, pp.153-162, 2003. 